\title{
Cosmology of Einstein-Vlasov system in a weak modification of general relativity
}

\section{Christian Corda}

June 8, 2021

\author{
International Institute for Theoretical Physics and Mathematics \\ Einstein-Galilei, via Santa Gonda 14, Prato Italy and Institute for Basic \\ Research, P. O. Box 1577, Palm Harbor, FL 34682, USA \\ E-mail address: cordac.galilei@gmail.com
}

\begin{abstract}
In earlier work it was shown that a weak modification of general relativity, in the linearized approach, renders a spherically symmetric and stationary model of the Universe. This was due to the presence of a third mode of polarization in the linearized gravity in which a "curvature energy" term is present. Such term was identified as the Dark Energy of the Universe.

In this letter a more realistic model is discussed. A different cosmological solution to the Einstein-Vlasov System is analysed. This solution shows reasonable results which are within the standard bounds predicted by the cosmological observations.
\end{abstract}

PACS numbers: 04.50.Kd, 98.80.Jk.

Over the past century, a standard cosmological model has emerged. With relatively few parameters, the model describes the evolution of the Universe and astronomical observations on scales ranging from a few to thousands of Megaparsecs. In this model the Universe is spatially flat, homogeneous and isotropic on large scales, composed of radiation, ordinary matter (electrons, protons, neutrons and neutrinos), non-baryon Cold Dark Matter, and Dark Energy. The galaxies and the large-scale structures grew gravitationally from tiny, nearly scale-invariant adiabatic Gaussian fluctuations [1, 2]. The WMAP data offers a demanding quantitative test of this model [3]. In other words, the Universe is seen like a dynamic and thermodynamic system. The test masses (i.e. the "particles") are the galaxies which are stellar systems with a number of the order of $10^{9}-10^{11}$ stars. The galaxies are located in clusters and super 
clusters, and observations show that, on cosmological scales, their distribution is uniform. These assumptions can be summarized in the Cosmological Principle: the Universe is homogeneous everywhere and isotropic around every point [1, 2]. The Cosmological Principle simplifies the analysis of the large scale structure. It implies that the proper distances between any two galaxies is given by a universal scale factor which is the same for any couple of galaxies [1, 2].

On the other hand, although the standard model of the Universe achieved great success, it also displayed many shortcomings and flaws. As a matter of fact, the accelerated expansion of the Universe, which is observed today, implies that cosmological dynamics is dominated by the so called Dark Energy, which gives a large negative pressure. This is the standard picture, in which this new ingredient is considered as a source on the right-hand side of the field equations. It should be some form of un-clustered, non-zero vacuum energy which, together with the clustered Dark Matter, drives the global dynamics. This is the so called "concordance model" $(\Lambda \mathrm{CDM})$ which gives, in agreement with the CMBR, LSS and SNeIa data, a good picture of the observed Universe today, but presents several shortcomings such as the well known "coincidence" and "cosmological constant" problems [4. An alternative approach is changing the left-hand side of the field equations, to see if the observed cosmic dynamics can be achieved by extending General Relativity [5]. In this different context, it is not required to find candidates for Dark Energy and Dark Matter, that, till now, have not been found. Only the "observed" ingredients, which are curvature and baryon matter, have to be taken into account. Considering this point of view, one can think that gravity is different at various scales and there is room for alternative theories. In principle, the most popular Dark Energy and Dark Matter models can be achieved considering $f(R)$ Theories of Gravity, where $R$ is the spacetime curvature, see the review [5], the recent results [6]-10] and references within. In this picture, the sensitive detectors for gravitational waves (GWs), like bars and interferometers, whose data analysis recently started [11, could, in principle, be important. In fact, a consistent GW astronomy will be the definitive test for General Relativity or, alternatively, a strong endorsement for Extended Theories of Gravity [12.

In earlier work [13] it was shown that a weak modification of General Relativity, in the linearized approach, renders a spherically symmetric and stationary model of the Universe. This was due to the presence of a third mode of polarization in the linearized gravity in which a "curvature energy" term is present. Such term was identified as the Dark Energy of the Universe.

In this letter a more realistic model is discussed. A different cosmological solution to the Einstein-Vlasov System is analysed. This solution shows reasonable results which are within the standard bounds predicted by the cosmological observations.

Let us consider the action

$$
S=\int d^{4} x \sqrt{-g} f_{0} R^{1+\varepsilon}+\mathcal{L}_{m}
$$

This is a particular choice in $f(R)$ Theories of Gravity [5]-[10]. 
In the limit $\varepsilon \rightarrow 0$ and $f_{0}=1$ the action (11) recovers the canonical form of the Einstein-Hilbert action of General Relativity [1, 2, i.e.

$$
S=\int d^{4} x \sqrt{-g} R+\mathcal{L}_{m}
$$

Criticisms on $f(R)$ Theories of Gravity arise from the fact that lots of such theories can be excluded by requirements of Cosmology and Solar System tests 14. However, in the case of the action (11), the discrepancy with respect to standard General Relativity is very weak, because $\varepsilon$ is a very small real parameter. Thus, the mentioned constraints could, in principle, be satisfied. In particular the authors of [14] found

$$
0 \leq \varepsilon \leq 7.2 * 10^{-19} .
$$

Fundamental constrains can be renormalized in order to obtain $f_{0}=1$ in the action (1)

We analyse interactions at cosmological scales, therefore the linearized theory in vacuum, i.e. with $\mathcal{L}_{m}=0$, can be considered. It gives a better approximation than the Newtonian theory [1, 2, 13. Thus, let us start from the pure curvature action

$$
S=\int d^{4} x \sqrt{-g} f_{0} R^{1+\varepsilon} .
$$

The theory arising from such an action has been linearized in [13. A short review of the linearized approach is needed for a better understanding of the theoretical framework.

By varying the action (4) with respect to $g_{\mu \nu}$, the field equations are obtained (through this letter the convention $G=1, c=1$ and $\hbar=1$ will be used and Greek indices run from 0 to 3$)$ ) 13 .

$$
G_{\mu \nu}=\frac{1}{(1+\varepsilon) f_{0} R^{\varepsilon}}\left\{-\frac{1}{2} g_{\mu \nu} \varepsilon f_{0} R^{1+\varepsilon}+\left[(1+\varepsilon) f_{0} R^{\varepsilon}\right]_{; \mu ; \nu}-g_{\mu \nu} \square\left[(1+\varepsilon) f_{0} R^{\varepsilon}\right]\right\} .
$$

The trace of these field equations is

$$
\square(1+\varepsilon) f_{0} R^{\varepsilon}=\frac{(1-\varepsilon)}{3} f_{0} R^{1+\varepsilon} .
$$

By making the identifications 13

$$
\Phi \rightarrow(1+\varepsilon) f_{0} R^{\varepsilon} \quad \text { and } \quad \frac{d V}{d \Phi} \rightarrow \frac{(1-\varepsilon)}{3} f_{0} R^{1+\varepsilon}
$$

a Klein - Gordon equation for the effective $\Phi$ scalar field is obtained. It can be written as

$$
\square \Phi=\frac{d V}{d \Phi} .
$$

Let us consider a little perturbation of the background, which is assumed given by a near Minkowskian background, i.e. a Minkowskian background plus 
$\Phi=\Phi_{0}$ (the Ricci scalar is assumed constant in the background) [13]. We also assume $\Phi_{0}$ to be a minimum for the effective potential $V$ :

$$
V \simeq \frac{1}{2} \alpha \delta \Phi^{2} \Rightarrow \frac{d V}{d \Phi} \simeq E^{2} \delta \Phi,
$$

and the constant $E$ has mass-energy dimension.

Let us write

$$
\begin{gathered}
g_{\mu \nu}=\eta_{\mu \nu}+h_{\mu \nu} \\
\Phi=\Phi_{0}+\delta \Phi .
\end{gathered}
$$

To first order in $h_{\mu \nu}$ and $\delta \Phi$, calling $\widetilde{R}_{\mu \nu \rho \sigma}, \widetilde{R}_{\mu \nu}$ and $\widetilde{R}$ the linearized quantity which correspond to $R_{\mu \nu \rho \sigma}, R_{\mu \nu}$ and $R$, the linearized field equations are 13

$$
\begin{gathered}
\widetilde{R}_{\mu \nu}-\frac{\widetilde{R}}{2} \eta_{\mu \nu}=\left(\partial_{\mu} \partial_{\nu} h_{\varepsilon}-\eta_{\mu \nu} \square h_{\varepsilon}\right) \\
\square h_{\varepsilon}=E^{2} h_{\varepsilon},
\end{gathered}
$$

where

$$
h_{\varepsilon} \equiv \frac{\delta \Phi}{\Phi_{0}}
$$

Notice that even if the minimum of the effective potential $V$ is the real number $0, V$ is not equal to $\Phi$, therefore we are not dividing by 0 in Eq. (12). In other words, as $V \neq \Phi$, the condition $V=0$ does not imply $\Phi_{0}=0$.

Then, from the second of Eqs. (11), one can define the mass-energy like [13]

$$
E \equiv \sqrt{\frac{\square h_{\varepsilon}}{h_{\varepsilon}}}=\sqrt{\frac{\square \delta \Phi}{\delta \Phi}}=\sqrt{\frac{\square \delta R^{\varepsilon}}{\delta R^{\varepsilon}}} .
$$

Thus, as the mass-energy is generated by variation of the Ricci scalar, we can say that, in a certain sense, it is generated by variation of spacetime curvature. This is exactly the "curvature energy" term [13.

We recall that, in the present case, the theory is viable as the modification of General Relativity is very weak and in agreement with requirements of Cosmology and Solar System tests [14.

In [13] it has been shown that, by using gauge transformations, the linearization process gives

$$
h_{\mu \nu}(t, z)=A^{+}(t-z) e_{\mu \nu}^{(+)}+A^{\times}(t-z) e_{\mu \nu}^{(\times)}+h_{\varepsilon}(t, z) \eta_{\mu \nu} .
$$

The term $A^{+}(t-z) e_{\mu \nu}^{(+)}+A^{\times}(t-z) e_{\mu \nu}^{(\times)}$describes the two standard polarizations of gravitational waves which arise from General Relativity, while the term $h_{\varepsilon}(t, z) \eta_{\mu \nu}$ is the massive field arising from the high order theory. In other words, the function $R^{\varepsilon}$ of the Ricci scalar generates a third polarization 
state for GWs which is not present in standard General Relativity. This third polarization has a "curvature" energy $E$, see [13 for details.

In the simple model of Universe in [13] the dynamic of the matter is described by the Vlasov equation [13, 15. The gravitational forces between the particles, i.e., the galaxies, are supposed to be mediated by the third mode of Eq. (14) after making the assumption that at cosmological scales such a mode becomes dominant (i.e. $A^{+}, A^{-} \ll h_{\varepsilon}$ ) [13. In this way the "curvature" mass-energy $E$ can be identified as the Dark Energy of the Universe $\simeq 10^{-29} \mathrm{~g} / \mathrm{cm}^{3}[3]$.

The model in 13 realized a spherically symmetric and stationary model of the Universe. The present work analyses a different cosmological solution to the Einstein-Vlasov System. The new model shows reasonable results which are within the standard bounds predicted by the cosmological observations.

In the hypothesis $A^{+}, A^{-} \ll h_{\varepsilon}$, Eq. (14) can be rewritten as

$$
h_{\mu \nu}(t, z)=h_{\varepsilon}(t, z) \eta_{\mu \nu}
$$

and the line element of the model is the conformally flat one

$$
d s^{2}=\left[1+h_{\varepsilon}(t, z)\right]\left(d t^{2}-d z^{2}-d x^{2}-d y^{2}\right) .
$$

By defining

$$
a^{2} \equiv 1+h_{\varepsilon}(t, z),
$$

the line element (16) results similar to the cosmological Friedmann-RobertsonWalker (FRW) line element of the standard homogeneous, isotropic and flat Universe [1, 2]

$$
d s^{2}=\left[a^{2}(t, z)\right]\left(+d t^{2}-d z^{2}-d x^{2}-d y^{2}\right) .
$$

Strictly speaking, this metric does not describe an homogeneous and isotropic universe, as $h_{\varepsilon}$ is also a function of $\mathrm{z}$. Thus, we have to further assume $\partial_{z} h_{\varepsilon}=0$, which removes the z-dependence [16.

To satisfy the condition demanding that the particles make up an ensemble with no collisions in the spacetime, the particle density must be a solution of the Vlasov equation

$$
\partial_{t} f+\frac{p^{a}}{p^{0}} \partial_{x^{a}} f-\Gamma_{\mu \nu}^{a} \frac{p^{\mu} p^{\nu}}{p^{0}} \partial_{p^{a}} f=0 .
$$

Here $\Gamma_{\mu \nu}^{\alpha}$ represent the usual connections, $f$ is the particle density and $p^{0}$ is determined by $p^{a}(a=1,2,3)$ according to the relation

$$
g_{\mu \nu} p^{\mu} p^{\nu}=-1
$$

13. 15, which expresses the condition that the four momentum $p^{\mu}$ lies on the mass shell of the metric.

A clarification is needed. We have to distinguish between the standard kinetic theory which is based upon Boltzmann equation [17 and the Einstein-Vlasov 
system which we are discussing in the present work. In a kinetic description the time evolution of the system is determined by the interactions between the particles, which depend on the physical situation [18]. For instance, the driving mechanism for the time evolution of a neutral gas is the collision between particles (the Boltzmann equation) 18. For a plasma the interaction is through the electromagnetic field produced by the charges (the Vlasov-Maxwell system), and in astrophysics the interaction between collisionless particles is gravitational (the Vlasov-Poisson system and the Einstein-Vlasov system) [18. Therefore, an important difference between the standard kinetic theory and the Einstein-Vlasov system which we are discussing in this letter is that in the first case there are collisions between particles while in the Einstein-Vlasov system particles interact gravitationally only and they are collisionless.

We recall that, in general, the Vlasov- Poisson system is [13, 15]

$$
\begin{gathered}
\partial_{t} f+v \cdot \nabla_{x} f-\nabla_{x} U \cdot \nabla_{v} f=0 \\
\triangle U=4 \pi \rho \\
\rho(t, x)=\int d v f(t, x, v),
\end{gathered}
$$

where $t$ denotes the time and $x$ and $v$ the position and the velocity of the galaxies. The function $U=U(t, x)$ is the average Newtonian potential generated by the galaxies. This system represents the non-relativistic kinetic model for an ensemble of particles with no collisions, which interacts through the gravitational forces that they generate collectively [13, 15. Thus, one can use such a system to describe the motion of galaxies within the Universe, thought of as pointlike particles, when the relativistic effects are negligible [13, 15. In this approach, the function $f(t, x, v)$ in the Vlasov- Poisson system (21) is non-negative and gives the density on phase space of the galaxies within the Universe.

By using the classical transformation from conformal time to synchronous time 1

$$
d t \rightarrow \frac{d t}{\sqrt{1+h_{\varepsilon}(t)}}
$$

the line element (16), in spherical coordinates, becomes

$$
d s^{2}=d t^{2}-\left[1+h_{\varepsilon}(t)\right]\left(d r^{2}+r^{2}\left(d \theta^{2}+\sin ^{2} \theta d \varphi^{2}\right)\right) .
$$

The metric tensor has the form

$$
g_{\mu \nu}=\left(\begin{array}{cc}
1 & 0 \\
0 & \gamma_{m n}
\end{array}\right),
$$

where $\gamma_{m n}=1+h_{\varepsilon}(t)$. 
Following [2], we define $\chi_{m n} \equiv \frac{\partial}{\partial t} \gamma_{m n}$. The Einstein field equations in the synchronous frame are:

$$
\begin{gathered}
R_{0}^{0}=-\frac{1}{2} \frac{\partial}{\partial t} \chi_{a}^{a}-\frac{1}{4} \chi_{a}^{b} \chi_{b}^{a}=\left(T_{0}^{0}-\frac{1}{2} T\right) \\
R_{a}^{0}=\frac{1}{2}\left(\chi_{a ; b}^{b}-\chi_{b ; a}^{a}\right)=T_{a}^{0} \\
R_{a}^{b}=-P_{a}^{b}-\frac{1}{2 \sqrt{\gamma}} \frac{\partial}{\partial t}\left(\sqrt{\gamma} \chi_{a}^{b}\right)=T_{a}^{b}-\frac{1}{2} \delta_{a}^{b} T,
\end{gathered}
$$

where $P_{a}^{b}$ is the Ricci tensor in 3 dimensions 2 .

On the other hand, the Einstein field equations in the Einstein-Vlasov system are 13,15

$$
G_{\mu \nu}=\frac{2}{\sqrt{-g}} \int f(t, x, p) p_{\mu} p_{\nu} \delta\left(p^{2}+m^{2}\right) d^{4} p,
$$

where $m$ is the mass of a particle (galaxy).

We can split the function $f(t, x, p)$ into a couple of equations for $f_{+}(t, x, p)$ and $f_{-}(t, x, p)$ which are constructed by reducing $f(t, x, p)$ respectively on the "upper" half and on the "lower" half of the mass shell. Eq. (19) becomes

$$
\partial_{t} f_{ \pm}=-\frac{1}{p_{ \pm}^{0}}\left(\gamma^{m n} p_{n} \frac{\partial}{\partial x_{m}}-\frac{1}{2} \frac{\partial \gamma^{n r}}{\partial x_{m}} p_{n} p_{r} \frac{\partial}{\partial p_{m}}\right) f_{ \pm} .
$$

Eq. (29) can be interpreted in Hamiltonian terms:

$$
p_{ \pm}^{0} \partial_{t} f_{ \pm}=\left\{H, f_{ \pm}\right\}
$$

where the Hamiltonian function is

$$
H \equiv \frac{1}{2} \gamma^{m n} p_{m} p_{n}
$$

One can calculate the components of energy-momentum tensor $T_{\mu \nu}$ in the approximation which considers galaxies like massless particles $(m=0$ in Eq. (28) )

$$
\begin{gathered}
T_{00}=\frac{1}{\left(\sqrt{1+h_{\varepsilon}(t)}\right)^{3} r^{2} \sin \theta} \int \frac{f_{+}+f_{-}}{\sqrt{1+h_{\varepsilon}(t)}} \sqrt{\frac{p_{1}^{2}+p_{2}^{2}}{r^{2}}+\frac{p_{3}^{2}}{r^{2} \sin \theta}} d^{3} p \\
T_{m n}=\frac{1}{\left(\sqrt{1+h_{\varepsilon}(t)}\right)^{3} r^{2} \sin \theta} \int \sqrt{1+h_{\varepsilon}(t)} \frac{\left(f_{+}+f_{-}\right)}{\sqrt{\frac{p_{1}^{2}+p_{2}^{2}}{r^{2}}+\frac{p_{3}^{2}}{r^{2} \sin \theta}}} p_{m} p_{n} d^{3} p \\
T_{0 m}=\frac{1}{\left(\sqrt{1+h_{\varepsilon}(t)}\right)^{3} r^{2} \sin ^{2} \theta} \int\left(f_{+}-f_{-}\right) p_{m} d^{3} p .
\end{gathered}
$$


The Einstein field equations (25), (26) and (27) give two independent dynamic equations which can be written down in terms of the scale factor $a=$ $\sqrt{1+h_{\varepsilon}(t)}$ :

$$
\begin{gathered}
\dot{a}^{2}=-1+\frac{1}{3 a} \int\left(f_{+}(s)+f_{-}(s)\right) \frac{s}{a} d^{3} s \\
\ddot{a}=-\frac{2}{a}-2 \frac{\dot{a}^{2}}{a}+\frac{1}{a^{2}} \int\left(f_{+}(s)+f_{-}(s)\right) d^{3} s,
\end{gathered}
$$

where

$$
s \equiv p_{1}^{2}+\frac{p_{2}^{2}}{r^{2}}+\frac{p_{3}^{2}}{r^{2} \sin ^{2} \theta} .
$$

By introducing the dimensionless variables $\underline{r}$ and $\underline{t}$ we put

$$
\begin{gathered}
a=a_{0} \underline{r} \\
t=a_{0} \underline{t} \\
\underline{\dot{r}}=\frac{d \underline{r}}{d \underline{t}} \\
j=\frac{1}{3} a_{0}^{2} \rho_{0},
\end{gathered}
$$

where $a_{0}$ is the present value of the scale factor of the Universe.

Eq. (35) becomes

$$
\begin{gathered}
\underline{\dot{r}}^{2}=-1+\frac{j}{\underline{r}^{2}} \\
\underline{r}_{0}=1 .
\end{gathered}
$$

The solution of the system (39) is

$$
\underline{r}(\underline{t})=\sqrt{j-(\underline{t}-\sqrt{j-1})^{2}}
$$

if $j \geq 1$. Notice that expression (40) becomes imaginary for certain values of the parameter $\underline{t}$ [17]. Such a parameter represents the cosmic time normalized by the present value of the scale factor $a_{0}$, see Eq. (38). By choosing the origin of the cosmic time at the present era of the cosmological evolution, i.e. $t_{0}=0$, expression (40) is always real if $j \geq 1$.

Returning to the $(t, a)$ variables we get:

$$
a(t)=a_{0} \sqrt{\frac{a_{0}^{2} \rho_{0}}{3}-\left(\frac{t}{a_{0}}-\sqrt{\frac{a_{0}^{2} \rho_{0}}{3}}-1\right)^{2}} .
$$

The today's observed Hubble radius and the today's observed density of the Universe are respectively $[3] a_{0} \gtrsim 10^{28} \mathrm{~cm}$ and $\rho_{0} \approx 10^{-57} \mathrm{~cm}^{-2}$. Therefore $j \approx 1$.

By inserting these data in Eq. (41) we obtain a singularity at a time 
$t_{s} \approx-10^{10}$ seconds and a value for the today's theoretical Hubble constant $H_{0}=\frac{\dot{a}_{0}}{a_{0}} \approx 10^{-29} \mathrm{~cm}^{-1}$.

As it follows from the above analysis, even under the assumption to neglect the baryon mass of the galaxies the results look reasonable. They are of the same order of magnitude of the standard cosmological model [3].

\section{Conclusion remarks}

In earlier work it was shown that a weak modification of general relativity, in the linearized approach, renders a spherically symmetric and stationary model of the Universe. This was due to the presence of a third mode of polarization in the linearized gravity in which a "curvature energy" term is present. Such term was identified as the Dark Energy of the Universe.

In this letter a more realistic model has been discussed. A different cosmological solution to the Einstein-Vlasov System has been analysed. This solution shows reasonable results which are within the standard bounds predicted by the cosmological observations.

We hope in further analyses which could insert the baryon mass too and realize a better fine-tuning the model with the cosmological observations.

\section{Acknowledgements}

The Ruggero M. Santilli Foundation has to be thanked for supporting this letter (Grant Number RMS-TH-5735A2310).

I strongly thank an unknown referee for useful comments and advices which permitted to improve this letter.

\section{References}

[1] C. W. Misner, K. S. Thorne and J. A. Wheeler, "Gravitation", W.H.Feeman and Company (1973).

[2] L. Landau L and E. Lifsits, Classical Theory of Fields (3rd ed.). London: Pergamon. ISBN 0-08-016019-0. Vol. 2 of the Course of Theoretical Physics (1971).

[3] C. L. Bennet et al., Astrophys. J. Suppl. 148, 1 (2003).

[4] P. J. E. Peebles and B. Ratra, Rev. Mod. Phys. 758559 (2003).

[5] S. Nojiri and S.D. Odintsov, Int. J. Geom. Meth. Mod. Phys. 4, 115-146 (2007).

[6] E. Elizalde, S. Nojiri, S. D. Odintsov, D. Saez-Gomez, arXiv:1006.3387 (2010).

[7] K. Bamba, S. Nojiri and S. D. Odintsov, J. Cosmol. Astropart. Phys. JCAP10(2008)045. 
[8] E. Elizalde, P. J. Silva, Phys. Rev. D78, 061501 (2008).

[9] G. Cognola, E. Elizalde, S. Nojiri, S.D. Odintsov and S. Zerbini, Phys. Rev. D 73, 084007 (2006).

[10] S. Nojiri and S. D. Odintsov, Phys. Rev. D 77026007 (2008).

[11] The LIGO Scientific Collaboration, Class. Quant. Grav. 26, 114013 (2009).

[12] C. Corda, Int. Journ. Mod. Phys. D, 18, 14, 2275-2282 (2009).

[13] C. Corda and H. Mosquera Cuesta, Europhys. Lett. 86, 20004 (2009).

[14] T. Clifton and J. D. Barrow, Phys. Rev. D 72, 103005 (2005).

[15] G. Rein and A. D. Rendall, Comm. Math. Phys. 150, 561- 83 (1992).

[16] C. Corda, Gen. Rel. Grav. 40, 2201-2212 (2008).

[17] Private communication with an unknown referee.

[18] H. Andréasson, Living Rev. Rel. 14, 4 (2011). 